\title{
New categorifications of the chromatic and dichromatic polynomials for graphs
}

\author{
by \\ Marko Stošić (Lisbon)
}

\begin{abstract}
For each graph $G$, we define a chain complex of graded modules over the ring of polynomials whose graded Euler characteristic is equal to the chromatic polynomial of $G$. Furthermore, we define a chain complex of doubly-graded modules whose (doubly) graded Euler characteristic is equal to the dichromatic polynomial of $G$. Both constructions use Koszul complexes, and are similar to the new Khovanov-Rozansky categorifications of the HOMFLYPT polynomial. We also give a simplified definition of this triply-graded link homology theory.
\end{abstract}

1. Introduction. In [3] Khovanov introduced the concept of categorification of the Jones polynomial for links. For each link $L$ in $S^{3}$ he defined a graded chain complex, with grading preserving differentials, whose graded Euler characteristic is equal to the Jones polynomial of the link $L$. This is done by starting from the state-sum expression for the Jones polynomial (which is written as an alternating sum), then constructing for each term a module whose graded dimension is equal to the value of that term, and finally, constructing the differentials as appropriate grading preserving maps, so that the complex obtained is a link invariant.

Using similar techniques by starting from the state-sum expression of the chromatic polynomial for graphs, the authors of [2] defined, for every graph $G$, a chain complex of graded modules whose Euler characteristic is the chromatic polynomial of $G$. In [11] the present author defined an infinite series of chain complexes of graded modules (one for each $n \in \mathbb{N}$ ) whose Euler characteristics are specializations of the two-variable dichromatic polynomial (and consequently the Tutte polynomial) of $G$. The specializations

2000 Mathematics Subject Classification: Primary 57M25.

Key words and phrases: graph, homology, chromatic polynomial, dichromatic polynomial, Koszul complex, Khovanov-Rozansky.

The author is supported by Fundação para a Ciência e a Tecnologia/(FCT), grant no. SFRH/BD/6783/2001. 
appear since we want to categorify a two-variable polynomial and the "standard" techniques of categorifying link (and graph) polynomials (see e.g. [7], [5], [4], [1], [3]) work only for one-variable polynomials.

In this paper we define a chain complex of doubly-graded modules whose doubly-graded Euler characteristic is equal to the whole two-variable dichromatic polynomial. The idea is partially inspired by the new version of categorification of the HOMFLYPT polynomial by Khovanov and Rozansky [8]. They defined a chain complex of doubly-graded modules whose doubly-graded Euler characteristic is equal to the whole two-variable HOMFLYPT polynomial. In Section 2 we describe a simplified version of their construction (this construction is also implicit in [6]).

Also, we give a new categorification of the chromatic polynomial for graphs. We do this in a different way than in [2]. We define chain groups (direct sums of modules corresponding to the vertices of the cube of resolutions) as the cohomologies of certain chain complexes.

\section{Triply-graded link homology}

2.1. Introduction. In this section we introduce a parametrization of the HOMFLYPT polynomial that we will categorify. It is very similar to the one in [8]. Throughout this section we will consider only braid diagrams $D$ of a link $L$, i.e. regular diagrams which are the closures of (upwards) oriented braids.

As is well known, every link can be represented by a braid diagram. Also, the closures of two braid diagrams $D_{1}$ and $D_{2}$ are isotopic as oriented links if and only if $D_{1}$ and $D_{2}$ are related by a sequence of Markov moves, which are the following (see [9]):

(i) conjugation: $D D^{\prime} \leftrightarrow D^{\prime} D$,

(ii) transformations in the braid group:

$$
\begin{aligned}
D & \leftrightarrow D \sigma_{i} \sigma_{i}^{-1}, \\
D & \leftrightarrow D \sigma_{i}^{-1} \sigma_{i}, \\
D \sigma_{j} \sigma_{i} & \leftrightarrow D \sigma_{i} \sigma_{j}, \quad|i-j|>1, \\
D \sigma_{i} \sigma_{i+1} \sigma_{i} & \leftrightarrow D \sigma_{i+1} \sigma_{i} \sigma_{i+1},
\end{aligned}
$$

(iii) transformations $D \leftrightarrow D \sigma_{n}^{ \pm 1}$, for a braid $D$ with $n$ strands.

In order to define the HOMFLYPT polynomial for a link $L$ from its braid diagram representation $D$, we will introduce a function $F$ on braid diagrams with values in the ring of rational functions in $q$ and $t$ defined uniquely by the following axioms:

- $F\left(D_{1}\right)=F\left(D_{2}\right)$ if $D_{1}, D_{2}$ are related by a Markov move (i).

- $F\left(D_{1}\right)=F\left(D_{2}\right)$ if $D_{1}, D_{2}$ are related by Markov moves (ii). 
- $F\left(D \sigma_{n}\right)=F(D)$ if the braid $D$ has $n$ strands.

- $F\left(D \sigma_{n}^{-1}\right)=-t^{-1} q^{-1} F(D)$ if the braid $D$ has $n$ strands.

- Skein relation: for every braid diagram $D$ with $n$ strands and $0<i<n$,

$$
q^{-1} F\left(D \sigma_{i}\right)-q F\left(D \sigma_{i}^{-1}\right)=\left(q^{-1}-q\right) F(D) .
$$

- If $U$ is the one-strand diagram of the unknot then $F(U)=1$.

In order to obtain a link invariant we need to normalize the function $F$. Let $\alpha=-t^{-1} q^{-1}$ and let

$$
G(D)=\sqrt{\alpha}^{n_{+}(D)-n_{-}(D)-s(D)+1} F(D),
$$

where $n_{+}(D), n_{-}(D)$ and $s(D)$ are the numbers of positive crossings, negative crossings and strands of $D$, respectively. We set

$$
\omega(D)=n_{+}(D)-n_{-}(D)-s(D)+1 .
$$

Obviously $G(D)$ is invariant under all Markov moves of braids and it satisfies the HOMFLYPT skein relation

$$
(q \sqrt{\alpha})^{-1} G\left(D \sigma_{i}\right)-q \sqrt{\alpha} G\left(D \sigma_{i}^{-1}\right)=\left(q^{-1}-q\right) G(D) .
$$

Hence, $G(D)$ is equal to the HOMFLYPT polynomial of the link $L$, normalized so that $G(U)=1$. In Section 2.3, we will define a triply-graded chain complex $\mathcal{C}(D)$ whose Euler characteristic is equal to $F(D)$.

First of all note our slightly different convention compared to [8] on the value of the unknot. This has the advantage that we can obtain the Alexander polynomial directly by specializing $t$ and $q(t=-q)$, and the whole sequence of $n$-specializations of the (reduced) HOMFLYPT polynomial (see [7], [10]). Specifically, by taking $t=-q^{1-2 n}$ we obtain polynomials $G_{n}(D)$ that satisfy the skein relation

$$
q^{-n} G_{n}\left(D \sigma_{i}\right)-q^{n} G_{n}\left(D \sigma_{i}^{-1}\right)=\left(q^{-1}-q\right) G_{n}(D),
$$

and whose value on the unknot is $G_{n}(U)=1$. Hence by suitably collapsing the tri-grading to a bi-grading we get a new categorification of the $n$ specializations of the HOMFLYPT polynomial.

2.2. Graphs with wide edges. In order to define the function $F(D)$ and hence the HOMFLYPT polynomial $G(D)$, we introduce trivalent graphs with wide edges as resolutions of crossings. Apart from the crossings $\sigma_{i}$ and $\sigma_{i}^{-1}$, we introduce wide edges $\bar{E}_{i}$ placed between the $i$ th and $(i+1)$ th strand of the braid, as in the following picture:

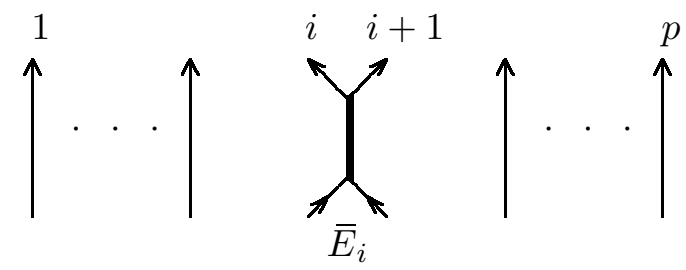


Then we can define the function $F(D)$ by resolving the crossings by using the following two relations:

$$
\begin{aligned}
F\left(D \sigma_{i}\right) & =F\left(D \bar{E}_{i}\right)-q^{2} F(D), \\
F\left(D \sigma_{i}{ }^{-1}\right) & =q^{-2} F\left(D \bar{E}_{i}\right)-q^{-2} F(D) .
\end{aligned}
$$

Here by $F(D)$ we mean the value of the function $F$ on the diagram that is the closure of the braid diagram $D$, and we have extended the domain of $F$ to include trivalent graphs. Then $F$ (restricted to braid diagrams) will satisfy the axioms from the previous subsection if and only if the values of $F$ on completely resolved trivalent graphs satisfy

$$
\begin{gathered}
F(U)=1, \\
F(D \cup U)=\frac{1+t^{-1} q}{1-q^{2}} F(D) \quad \text { if } D \text { is not an empty diagram, } \\
F\left(D \bar{E}_{n}\right)=\frac{1+t^{-1} q^{3}}{1-q^{2}} F(D) \quad \text { if } D \text { is a diagram with } n \text { strands, } \\
F\left(D \bar{E}_{i}^{2}\right)=\left(1+q^{2}\right) F\left(D \bar{E}_{i}\right), \\
F\left(D \bar{E}_{i} \bar{E}_{i+1} \bar{E}_{i}\right)+q^{2} F\left(D \bar{E}_{i+1}\right)=F\left(D \bar{E}_{i+1} \bar{E}_{i} \bar{E}_{i+1}\right)+q^{2} F\left(D \bar{E}_{i}\right) .
\end{gathered}
$$

We will use (2) and (3) in resolving the crossings in the definition of the triply-graded chain complex that categorifies the HOMFLYPT polynomial.

2.3. Categorification of the two-variable HOMFLYPT polynomial. In this subsection we will give an alternative, simpler and (essentially) equivalent construction to the one in [8] (a similar simplification is also implicit in $[6])$.

Essentially, we will set the variable $a$ from [8] to be 0, but we will keep the double grading of the ring of polynomials $R^{\prime}=\mathbb{Q}\left[x_{1}, \ldots, x_{2 n}\right]$. Specifically, to every arc (line between two crossings) we will assign a different variable $x_{i}$, $i=1, \ldots, 2 n$, where $n$ is the number of crossings of a given planar projection $D$ of a knot $K$. We define the bidegree of every $x_{i}$ to be $(0,2)$ and we put the field of coefficients $\mathbb{Q}$ in bidegree $(0,0)$. Also, by $\{\cdot, \cdot\}$ we denote a shift in bigrading.

REMARK 1. Note that this corresponds to the case $n=-1$ in [7], but with the introduction of a new grading direction.

Let $L$ be a link and let $D$ be its braid diagram presentation. Let $I$ be the ideal of $R^{\prime}$ generated by the monomials $x_{1}+x_{2}-x_{3}-x_{4}$ for every crossing of $D$, and let $R=R^{\prime} / I$ (see the picture below for the notation). 
To each crossing we will assign 0 - and 1-resolutions according to the following picture:

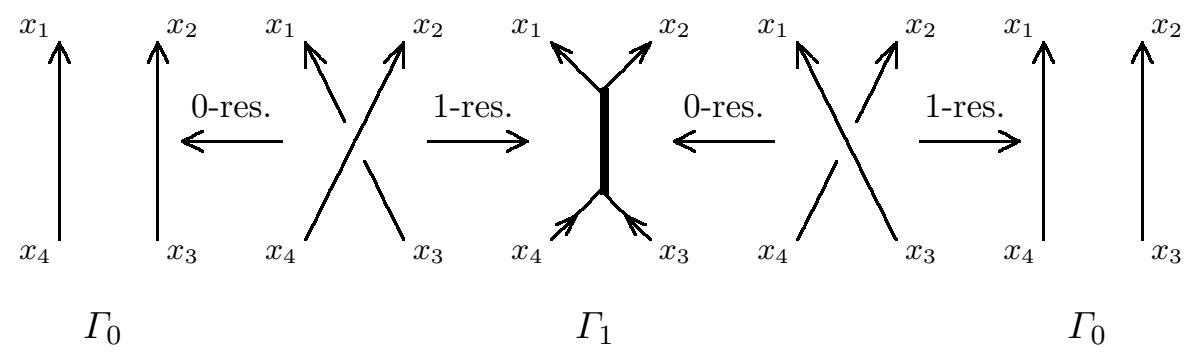

We call the resolutions obtained $\Gamma_{0}$ and $\Gamma_{1}$, respectively, according to the picture. To the resolution $\Gamma_{0}$ we assign the complex

$$
\mathcal{C}\left(\Gamma_{0}\right): \quad 0 \rightarrow R\{-1,1\} \stackrel{x_{2}-x_{3}}{\longrightarrow} R \rightarrow 0
$$

and to the resolution $\Gamma_{1}$ (the one with the wide edge) the complex

$$
\mathcal{C}\left(\Gamma_{1}\right): \quad 0 \rightarrow R\{-1,3\} \stackrel{\left(x_{2}-x_{3}\right)\left(x_{4}-x_{2}\right)}{\longrightarrow} R \rightarrow 0 .
$$

Assume that there are no free circles in the diagram $D$. If we resolve all the crossings of $D$ we obtain a trivalent graph with wide edges. There are $2^{n}$ such resolutions $\Gamma$ of $D$ and to each we assign the tensor product of $\mathcal{C}\left(\Gamma_{0}\right)$ and $\mathcal{C}\left(\Gamma_{1}\right)$, over all crossings $c(D)$, depending on the type of resolution that appeared. In this way we obtain a complex $\mathcal{C}(\Gamma)$ and to each resolution $\Gamma$ we will assign its cohomology $H(\Gamma)=H(\mathcal{C}(\Gamma))$.

As in [8], we can show that $H(\Gamma)$ categorifies the relations (5)-(8). For example, the relation (5) becomes

$$
H(\Gamma \cup \text { unknot }) \cong\left(H(\Gamma) \otimes \mathbb{Q}\left[x_{i}\right]\right) \oplus\left(H(\Gamma) \otimes \mathbb{Q}\left[x_{i}\right]\{-1,1\}\right),
$$

where $x_{i}$ is the label assigned to the circle (unknot). Note that in all definitions only the differences $x_{i}-x_{j}$ appear. Thus we can work with the smaller ring of polynomials $R^{\prime \prime}=\mathbb{Q}\left[x_{2}-x_{1}, \ldots, x_{2 n}-x_{1}\right]$ instead of $R^{\prime}$ (as in [8]).

If we have free circles in $D$, we introduce a new variable $y$ with $\operatorname{deg} y=(0,2)$, extend the ring of polynomials to $R[y]$ and replace $R$ by $R[y]$ in the complexes $\mathcal{C}\left(\Gamma_{i}\right), i=0,1$. Finally, to every free circle we assign the complex

$$
0 \rightarrow R^{\prime}[y]\{-1,1\} \stackrel{y}{\rightarrow} R^{\prime}[y] \rightarrow 0,
$$

and we tensor these complexes with $\mathcal{C}(\Gamma)$. In this way we obtain a good value of the unknot (4), i.e. $H(U) \cong \mathbb{Q}$.

We again organize the $2^{n}$ total resolutions $\Gamma$ of the diagram $D$ in the same cubic complex as in the standard categorifications. To each vertex of the cube (i.e. to each total resolution $\Gamma$ ) we assign the graded vector space $H(\Gamma)$. 
We will introduce the differentials between those cohomology groups as the maps induced by the (grading preserving) homomorphisms between the corresponding complexes $\mathcal{C}(\Gamma)$. Since these complexes are built as the tensor products of $\mathcal{C}\left(\Gamma_{0}\right)$ and $\mathcal{C}\left(\Gamma_{1}\right)$ it is enough to specify the homomorphisms between these two complexes. For a positive crossing $c$ we define the following complex of complexes:

$$
\mathcal{C}_{c}: \quad 0 \rightarrow \mathcal{C}\left(\Gamma_{0}\right)\{0,2\} \stackrel{\chi_{0}}{\rightarrow} \mathcal{C}\left(\Gamma_{1}\right) \rightarrow 0,
$$

where $\mathcal{C}\left(\Gamma_{1}\right)$ is in cohomological degree 0 , and the map $\chi_{0}$ is given by

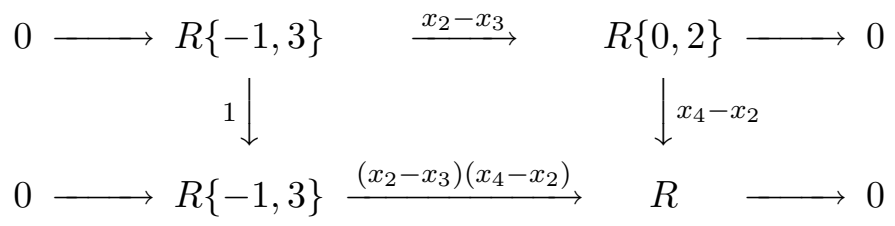

For a negative crossing $c$ we define the following complex of complexes:

$$
\mathcal{C}_{c}: \quad 0 \rightarrow \mathcal{C}\left(\Gamma_{1}\right)\{0,-2\} \stackrel{\chi_{1}}{\longrightarrow} \mathcal{C}\left(\Gamma_{0}\right)\{0,-2\} \rightarrow 0,
$$

where $\mathcal{C}\left(\Gamma_{1}\right)$ is in cohomological degree 0 , and the map $\chi_{1}$ is given by

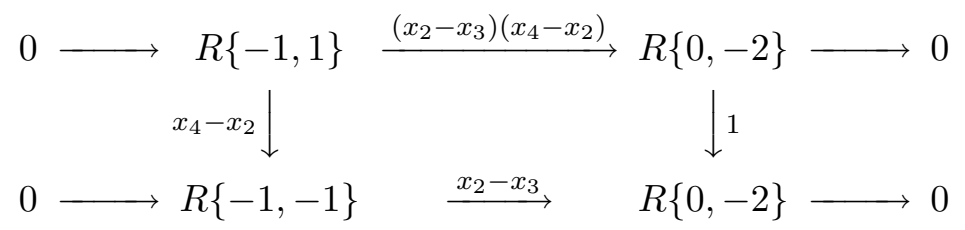

Define $\mathcal{C}(D)$ as the tensor product of $\mathcal{C}_{c}$ over all crossings $c(D)$. It is a complex built up from the Koszul complexes $\mathcal{C}(\Gamma)$, over all the total resolutions $\Gamma$ of the diagram $D$, and the differential preserves the bigrading of each term $\mathcal{C}^{j}(D)$. Every $\mathcal{C}^{j}(D)$ decomposes as a direct sum of contractible two-term complexes and its cohomology $H\left(\mathcal{C}^{j}(D)\right)$, which is denoted by $\mathcal{C} H^{j}(D)$. The differential induces grading preserving maps $\delta$ from $\mathcal{C} H^{j}(D)$ to $\mathcal{C} H^{j+1}(D)$ and we denote the complex obtained in this way by $\mathcal{C} H(D)$. The cohomology $H(D)=H(\mathcal{C} H(D), \delta)$ is triply-graded:

$$
H(D)=\bigoplus_{j, k, l} H_{k, l}^{j}(D) \text {. }
$$

Here $j$ is the cohomology degree, and $k$ and $l$ come from the internal bigrading of the chain groups.

In complete analogy with [8] we find that $H(D)$ does not depend on the choice of the braid presentation $D$ of a link $L$, up to an overall shift in the triple grading. Also, since $H(\Gamma)$ categorifies the relations (4)-(8) 
and since the differentials are induced by the grading preserving maps (9) and (10) which obviously categorify the relations (2) and (3), the bigraded Euler characteristic of $\mathcal{C H}(D)$ is equal to $F(D)$. Finally, by introducing half-integral shifts as in [12] (in order to compensate the powers of $\alpha$ from (1)) by

$$
\mathcal{H}(D)=H(D)[\omega(D) / 2]\{-\omega(D) / 2,-\omega(D) / 2\},
$$

we obtain a triply-graded homology theory, which does not depend on the choice of the braid presentation $D$ of the link $L$ and whose bi-graded Euler characteristic is equal to the two-variable HOMFLYPT polynomial of $L$.

\section{New categorifications of the chromatic and dichromatic polynomials for graphs}

3.1. Introduction. In this section we will define a complex of doublygraded modules whose doubly-graded Euler characteristic is equal to the whole two-variable dichromatic polynomial. The idea is partially inspired by the categorification of the HOMFLYPT polynomial described in the previous section. Also, we give a new categorification of the chromatic polynomial for graphs.

A graph $G$ is specified by a set of vertices $V(G)$ and a set of edges $E(G)$. If $e$ is an arbitrary edge of $G$, then by $G-e$ we denote the graph $G$ with the edge $e$ deleted, and by $G / e$ the graph obtained by contracting $e$ (i.e. by identifying the vertices incident to $e$ and deleting $e$ ).

3.2. The chromatic polynomial. If $q$ is a positive integer, the chromatic polynomial $P_{G}(q)$ is defined as the number of ways to color the vertices of $G$ by using at most $q$ colors, so that any two vertices which are connected by an edge receive different colors. It is well known that the chromatic polynomial can be equivalently defined by the following two axioms:

$$
\begin{aligned}
P_{G} & =P_{G-e}-P_{G / e}, \\
P_{N_{k}} & =q^{k},
\end{aligned}
$$

where $N_{k}$ is the graph with $k$ vertices and no edges. By using these axioms, we can obviously assume that the domain of the chromatic polynomial is the set of complex numbers. Furthermore, instead of $q$ in axiom $(C 2)$ we will put $1 /(1-q)$ (with $|q|<1)$.

By repeated use of $(C 1)$ (which is the famous deletion-contraction rule) we will obtain the value of the chromatic polynomial as a sum of contributions from all spanning subgraphs of $G$ (subgraphs that contain all vertices of $G$ ), which we will call states. Furthermore, if for each subset $s \subset E(G)$ we denote by $[G: s]$ the graph whose set of vertices is $V(G)$ and set of 
edges is $s$, then the contribution of the graph $[G: s]$ is $(-1)^{|s|}(1-q)^{-k(s)}$, where $|s|$ is the number of elements of $s$ and $k(s)$ is the number of connected components of $[G: s]$. Hence, we obtain the expression

$$
P_{G}(q)=\sum_{s \subset E(G)}(-1)^{|s|}(1-q)^{-k(s)}=\sum_{i \geq 0}(-1)^{i} \sum_{s \subset E(G),|s|=i}(1-q)^{-k(s)},
$$

which is called the state-sum expansion of the polynomial $P_{G}(q)$.

In Subsection 3.4 we will define a graded chain complex $\mathcal{C}(G)$ of modules whose graded Euler characteristic is equal to $P_{G}(q)$.

3.3. The dichromatic polynomial. The dichromatic polynomial $P_{G}(q, v)$ of the graph $G$ is a two-variable generalization of the chromatic polynomial given by the following two axioms:

$$
\begin{aligned}
P_{G} & =P_{G-e}-q P_{G / e}, \\
P_{N_{k}} & =v^{k},
\end{aligned}
$$

where $N_{k}$ is the graph with $k$ vertices and no edges.

From $(D 1)$ we have a recursive expression for the dichromatic polynomial in terms of the value of the polynomial on graphs with a smaller number of edges. Indeed, as in the case of the chromatic polynomial, the contribution of the state $[G: s]$ is $(-1)^{|s|} q^{|s|} v^{k(s)}$. Hence, we obtain the expression

$$
P_{G}(q, v)=\sum_{s \subset E(G)}(-1)^{|s|} q^{|s|} v^{k(s)}=\sum_{i \geq 0}(-1)^{i} q^{i} \sum_{s \subset E(G),|s|=i} v^{k(s)},
$$

which is called the state-sum expansion of the polynomial $P_{G}(q, v)$. However, we will use a slightly different parametrization, given by

$$
D_{G}(t, q)=\left(1+t^{-1} q\right)^{m} P_{G}\left(q, \frac{1+t^{-1} q}{1-q}\right)
$$

where $m$ is the number of edges of $G$.

In Subsection 3.5 we will define a chain complex $\mathcal{D}(G)$ of doubly-graded modules whose doubly-graded Euler characteristic is equal to $D_{G}(t, q)$.

3.4. The categorification of the chromatic polynomial. Let $n$ denote the number of vertices of the graph $G$. Let $R$ be the ring of polynomials in $n$ variables over $\mathbb{Q}$, i.e. $R=\mathbb{Q}\left[x_{1}, \ldots, x_{n}\right]$. We introduce a grading in $R$ by giving degree 1 to every $x_{i}$. Order the set of vertices of $G$ and assign the variable $x_{i}$ to the $i$ th vertex. Finally, to every edge $e \in E(G)$ with endpoints $i_{e}$ and $j_{e}$, assign the monomial $m_{e}=x_{i_{e}}-x_{j_{e}}$ (the ambiguity of the sign does not affect the later construction).

3.4.1. The cubic complex construction. Let $s \subset E(G)$ be a subset of the set of edges of $G$, and let $[G: s]$ be the corresponding state of $G$. Consider 
the ideal $I_{s}$ in $R$ generated by the monomials $m_{e}$ for all edges $e \in s$. Finally, to the state $[G: s]$ assign the module $R_{s}=R / I_{s}$.

Proposition 1. The quantum graded dimension of $R_{s}$ is equal to $(1-q)^{-k(s)}$, where $k(s)$ denotes the number of connected components of $[G: s]$.

Proof. Let $i$ and $j$ be arbitrary two vertices of $G$. They obviously belong to the same connected component of $[G: s]$ if and only if there exists a sequence of edges belonging to $s$ which connects $i$ and $j$, which obviously happens if and only if $x_{i}-x_{j}$ belongs to $I_{s}$. Hence, all the variables corresponding to the vertices from the same component must be the same in $R_{s}$. In other words, $R_{s}$ is isomorphic to the ring of polynomials (over $\mathbb{Q}$ ) in $k(s)$ variables, and hence

$$
q \operatorname{dim} R_{s}=\left(\sum_{i \geq 0} q^{i}\right)^{k(s)}=(1-q)^{-k(s)} .
$$

Denote by $m$ the number of edges of $G$, and fix an ordering on the set $E(G)$, denoted by $\left(e_{1}, \ldots, e_{m}\right)$. Now we define the chain complex $\mathcal{C}$ in a standard way, by "summing over columns" of our cubic complex: for each $i$ with $0 \leq i \leq m$, we define the $i$ th chain group $\mathcal{C}^{i}(G)$ as the direct sum of $R_{s}$ over all $s \subset E(G)$ such that $|s|=i$.

Now, let us turn to the differential. We define the map $d^{i}$ from $\mathcal{C}^{i}(G)$ to $\mathcal{C}^{i+1}(G)$ as a sum of maps between the direct summands of the chain groups. The only nonzero maps are the maps from $R_{s}$ to $R_{s \cup\{e\}}$ with $e \notin s$ (which are exactly the ones that correspond to the edges of the cube), and we set them (up to a sign) to be the identity (i.e. the map that sends $f+I_{s}$ to $f+I_{s \cup\{e\}}$ for every $f \in R$ ).

We now introduce signs in a standard way in order to make the cube anticommutative, and hence to make the square of the differential equal to 0 . Namely, we put minus signs exactly for those maps $R_{s} \rightarrow R_{s \cup\{e\}}$ with an odd number of edges in $s$ which are ordered before $e$.

In this way we have obtained a chain complex, $\mathcal{C}(G)$, of graded $R$ modules with grading preserving differential. Its homology groups obviously do not depend on the ordering of the vertices of $G$, and also do not depend on the ordering of the edges (as in [2, Section 2.2.3]), and hence we obtain

THEOREM 2. The homology groups of the chain complex $\mathcal{C}(G)$ are invariants of the graph $G$, and the graded Euler characteristic of $\mathcal{C}(G)$ is equal to the chromatic polynomial $P_{G}(q)$.

3.4.2. Alternative description. Now we will give an equivalent definition of the chain complex $\mathcal{C}(G)$ in terms of Koszul complexes. 
To each edge $e \in E(G)$ we assign two complexes, $\mathcal{C}_{e-}$ and $\mathcal{C}_{e+}$, defined in the following way:

$$
\begin{array}{ll}
\mathcal{C}_{e-}: & 0 \rightarrow R \stackrel{0}{\longrightarrow} R \rightarrow 0, \\
\mathcal{C}_{e+}: & 0 \rightarrow R \stackrel{x_{i}-x_{j}}{\longrightarrow} R \rightarrow 0,
\end{array}
$$

where $i$ and $j$ are the endpoints of $e$. Now, to every subset $s \subset E(G)$ we assign a complex $\mathcal{C}_{s}$ which is the tensor product of $\mathcal{C}_{e \pm}$, where we take + if $e \in s$ and - if $e \notin s$. Finally, to the state $[G: s]$ we assign the cohomology of $\mathcal{C}_{s}$ at the rightmost position.

To build the differentials, we introduce the (grading preserving) maps $d_{e}$ as the maps induced on cohomology by the following homomorphism from $\mathcal{C}_{e-}$ to $\mathcal{C}_{e+}$ :

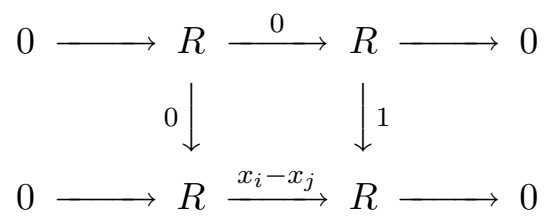

Here we put the upper row in cohomological degree 0 , and the lower one in cohomological degree 1.

Now, in order to define the differentials, just tensor all the chain complexes and maps (11) between them over all edges $e$ of $E(G)$. If we take the cohomology only at the rightmost position in each "horizontal" complex (the ones in the same cohomological degree with respect to the definition after (11)), and as the differentials are the induced maps between them, we obtain a complex $\mathcal{C}^{\prime}(G)$ which is isomorphic to the complex $\mathcal{C}(G)$ from the previous subsection.

3.5. The categorification of the dichromatic polynomial. In order to categorify the dichromatic polynomial we will have to introduce a new grading direction, and we will use the whole Koszul complex (actually a slightly modified one) that we have used in the previous subsection.

We order the vertices of $G$, and to the $i$ th one $(1 \leq i \leq n=\sharp V(G))$ we assign the variable $x_{i}$. We define the bidegree of all $x_{i}$ as $(0,1)$. Define the bigraded ring $R$ by $R=\mathbb{Q}\left[x_{1}, \ldots, x_{n}\right]$, where we put the field $\mathbb{Q}$ in bidegree $(0,0)$.

To each edge $e$ whose endpoints are the $i$ th and $j$ th vertex, we can associate two resolutions of the graph $G$ : the first one with the edge $e$ contracted (i.e. we identify the vertices $i$ and $j$ ), and the second one with the edge $e$ deleted. To the first resolution we assign the complex

$$
\mathcal{D}(e+): \quad 0 \rightarrow R\{-1,1\} \stackrel{x_{i}-x_{j}}{\longrightarrow} R \rightarrow 0,
$$


and to the second the complex

$$
\mathcal{D}(e-): \quad 0 \rightarrow R\{-1,1\} \stackrel{0}{\longrightarrow} R \rightarrow 0 .
$$

Let $s$ be an arbitrary subset of $E(G)$ and let $[G: s]$ be the corresponding state of $G$. To $[G: s]$ we assign the (Koszul) complex $\mathcal{D}^{\prime}(s)$ of bigraded $R$ modules obtained by tensoring the complexes $\mathcal{D}(e+)$, where $e$ runs over all edges in $s$, and $\mathcal{D}(f-)$, where $f$ runs over all edges in $E(G) \backslash s$. We denote its (bigraded) cohomology by $H^{\prime}(s)$ (the direct sum of the cohomology groups of $\left.\mathcal{D}^{\prime}(s)\right)$.

Proposition 2. The quantum bigraded dimension of $H^{\prime}(s)$ is equal to

$$
\left(1+t^{-1} q\right)^{m-n}\left(\frac{1+t^{-1} q}{1-q}\right)^{k(s)}
$$

where $k(s)$ is the number of connected components of $[G: s]$, and $n$ and $m$ are the numbers of vertices and edges of $G$, respectively.

Proof. As in the proof of Proposition 1, we find that the cohomology at the rightmost position of $\mathcal{D}^{\prime}(s)$ is isomorphic to the ring of polynomials in $k(s)$ variables. However, here we will also have the cohomology at the leftmost position in each of the $\mathcal{D}(e \pm)$, which is isomorphic to the same ring of polynomials in $k(s)$ variables, but shifted by the bidegree $\{-1,1\}$, for all $\mathcal{D}(e-)$ and for a certain number of the $\mathcal{D}(e+)$. We show by induction on $|s|$ that the total number of such $e$ 's, denoted by $c(s)$, is equal to $k(s)-n+m$.

If $|s|=0$ then we have the tensor product of $m$ complexes with all the mappings equal to zero, and hence the number of edges which contribute with nontrivial cohomology at the leftmost position is equal to $m=k(s)-$ $n+m$ (note that in this case $k(s)=n$ ). Now suppose that the formula is true for some subset $s$ and consider the state $[G:(s \cup e)]$ with $e \in E(G) \backslash s$. Denote the endpoints of $e$ by $i$ and $j$, and set $s^{\prime}=s \cup e$. This means that $\mathcal{D}^{\prime}\left(s^{\prime}\right)$ is formed by the tensor product of the same complexes as $\mathcal{D}^{\prime}(s)$ with $\mathcal{D}(e+)$ instead of $\mathcal{D}(e-)$. Now, $\mathcal{D}(e+)$ will have nontrivial cohomology at the leftmost position if and only if $x_{i}-x_{j}$ belongs to the ideal generated by the monomials defined by the edges of $s$, i.e. if and only if the vertices $i$ and $j$ belong to the same connected component of $[G: s]$. In other words, we have $c\left(s^{\prime}\right)=c(s)$ if $k\left(s^{\prime}\right)=k(s)$, and $c\left(s^{\prime}\right)=c(s)-1$ if $k\left(s^{\prime}\right)=k(s)-1$. So we have $c(s)=k(s)-n+m$ as desired.

Hence the total bigraded dimension of $H^{\prime}(s)$ is equal to

$$
\left(1+t^{-1} q\right)^{k(s)-n+m}(1-q)^{-k(s)} \text {. }
$$

Furthermore, to every vertex $v$ of the graph $G$, we assign the same complex as $\mathcal{D}(e-)$. Now, if we tensor these complexes over all vertices of $G$ and tensor the complex obtained with $\mathcal{D}^{\prime}(s)$, we obtain the complex $\mathcal{D}(s)$. We 
denote the cohomology of $\mathcal{D}(s)$ by $H(s)$, and that is the space we will assign to the state $[G: s]$. Obviously, we have

$$
q \operatorname{dim} H(s)=\left(1+t^{-1} q\right)^{m}\left(\frac{1+t^{-1} q}{1-q}\right)^{k(s)} .
$$

In order to introduce the differentials between the cohomologies $H(s)$, we will define the (grading preserving) homomorphism $d(e)$ from $\mathcal{D}(e+)\{0,1\}$ to $\mathcal{D}(e-)$, and then for the differentials we take the induced mappings on cohomology. We define $d(e)$ by

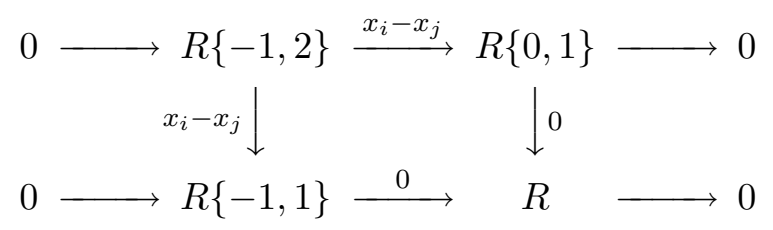

We put the upper row in cohomological degree -1 , and the lower row in cohomological degree 0 . We denote this complex of complexes by $\mathcal{D}_{e}$.

For the graph $G$ define the complex of (Koszul) complexes by tensoring $\mathcal{D}_{e}$ over all edges $e$ of $G$. By taking the cohomology $H^{j}(G),-m \leq j \leq 0$, in each "horizontal" complex and defining the differentials between them to be the ones induced by the tensor product of $d(e)$ 's, we obtain a triply-graded complex $\mathcal{D}(G)$.

From the definition we have

THEOREM 3. The homotopy class of the complex $\mathcal{D}(G)$ is an invariant of the graph $G$ whose bigraded Euler characteristic is equal to the dichromatic polynomial $D_{G}(t, q)$ of the graph $G$.

\section{References}

[1] D. Bar-Natan, Khovanov's homology for tangles and cobordisms, Geom. Topol. 9 (2005), 1443-1499.

[2] L. Helme-Guizon and Y. Rong, A categorification for the chromatic polynomial, Algebr. Geom. Topol. 5 (2005), 1365-1388.

[3] M. Khovanov, A categorification of the Jones polynomial, Duke Math. J. 101 (2000), $359-426$.

[4] - Categorifications of the colored Jones polynomial, arXiv:math.QA/0302060.

[5] —, sl(3) link homology, Algebr. Geom. Topol. 4 (2004), 1045-1081.

[6] - , Triply-graded link homology and Hochschild homology of Soergel bimodules, arXiv:math.GT/0510265.

[7] M. Khovanov and L. Rozansky, Matrix factorizations and link homology, arXiv: math.QA/0401268.

[8] —, - Matrix factorizations and link homology II, arXiv:math.QA/0505056.

[9] A. Markov, Über die freie Äquivalenz geschlossener Zöpfe, Mat. Sb. 1 (1935), 73-78.

[10] H. Murakami, T. Ohtsuki and S. Yamada, HOMFLY polynomial via an invariant of colored plane graphs, Enseign. Math. (2) 44 (1998), 325-360. 
[11] M. Stošić, Categorification of the dichromatic polynomial for graphs, arXiv:math. GT/0504239; J. Knot Theory Ramif., to appear.

[12] H. Wu, Braids, transversal knots and the Khovanov-Rozansky theory, arXiv:math. GT/0508064.

Departamento de Matemática and

CEMAT-Centro de Matemática e Aplicações

Instituto Superior Técnico

Av. Rovisco Pais 1

1049-001 Lisbon, Portugal

E-mail: mstosic@math.ist.utl.pt

Received 14 July 2005;

in revised form 2 February 2006 